\title{
Ein neuer Versuch mit Alternaria-Extrakten
}

\author{
Die Hyposensibilisierung mit Schimmelpilzextrakten wird aufgrund \\ widersprüchlicher Studienergebnisse zu Sicherheit und Wirksamkeit \\ kontrovers diskutiert. Ein spanisches Autorenteam testete jetzt ein \\ selbst hergestelltes Alternaria-alternata-Präparat.
}

\begin{abstract}
Alergologen aus Pamplona und Bilbao bezogen 28 Kinder und Jugendliche in ihre doppelblinde und plazebokontrollierte Studie ein. Bei den Probanden lag eine allergische Rhinitis oder allergisches Asthma bronchiale aufgrund einer Sensibilisierung gegen Alternaria alternata vor. Von den Studienteilnehmern erhielten 14 ein Jahr lang eine konventionelle Immuntherapie mit einem selbst hergestellten Extrakt aus $\mathrm{Al}$ ternaria alternata, die anderen 14 ein Plazebopräparat.
\end{abstract}

Insgesamt 23 Patienten beendeten die Studie. Während des gesamten Jahres trat nur in zwei Fällen - beide in der Verumgruppe - eine moderate Reaktion nach der Injektion auf. Der AsthmaSymptomscore hatte sich in der Verumgruppe nach sechs Monaten signifikant verbessert, in der Plazebogruppe war er unverändert geblieben. Die Symptomscores zu Rhinitis oder Konjunktivitis zeigten in beiden Gruppen dagegen keine signifikanten Veränderungen. Der Peak Expiratory Flow zeigte unter Im- muntherapie während des gesamten Beobachtungszeitraums signifikante Verbesserungen gegenüber den Ausgangswerten. Unter Plazebo waren hier keine signifikanten Veränderungen nachzuweisen. Der Medikationsscore war bereits zu Beginn der Studie in beiden Gruppen niedrig gewesen und es ergaben sich hier auch keine signifikanten Veränderungen.

Fazit: Die spezifische Immuntherapie mit Alternaria alternata war in dieser Studie sicher und in einigen Parametern signifikant besser als Plazebo. Untersuchungen mit höheren Fallzahlen sollten die Resultate bestätigen.

Tabar Al et al. Double-blind, placebo-controlled study of Alternaria alternata immunotherapy: clinical efficacy and safety. Pediatr Allergy Immunol 2008; 19: 67-75

\section{Hypoallergene Variante im Test}

\section{Bei jeder spezifischen Immuntherapie besteht die Gefahr einer aller- gischen Reaktion. Abhilfe könnten in Zukunft rekombinant herge- stellte und hypoallergene Präparate schaffen.}

$\mathrm{D}$ erzeit wird intensiv daran gearbeitet, die spezifische Immuntherapie durch modifizierte Allergene sicherer zu machen. Änderungen der Konformation sollen die Bindung von IgE-Antikörpern an die entsprechenden Epitope des Allergens erschweren, den Kontakt mit T-Zellen jedoch nicht beeinflussen. Ein deutsches Forscherteam untersuchte vor diesem Hintergrund eine rekombinant hergestellte Variante der 1.0101-Isoform von Bet v 1, die sich durch eine abweichende Faltungsvariante auszeichnet. Sie verglichen dieses rekombinante Allergen (rBet v 1-FV) mit dem natürlichen Allergen (nBet v 1) und einem rekombinanten Allergen mit normaler Faltstruktur (rBet v 1-WT).

In der Gelelektrophorese zeigten alle drei Allergene eine einheitliche Bande bei $17,5 \mathrm{kDa}$. Im Westernblot mit Serum von Birkenpollenallergikern konnte gezeigt werden, dass sich das natürliche Allergen und das rekombinante Allergen mit normaler Faltung deutlich an IgEAntikörper banden. Das Allergen mit der

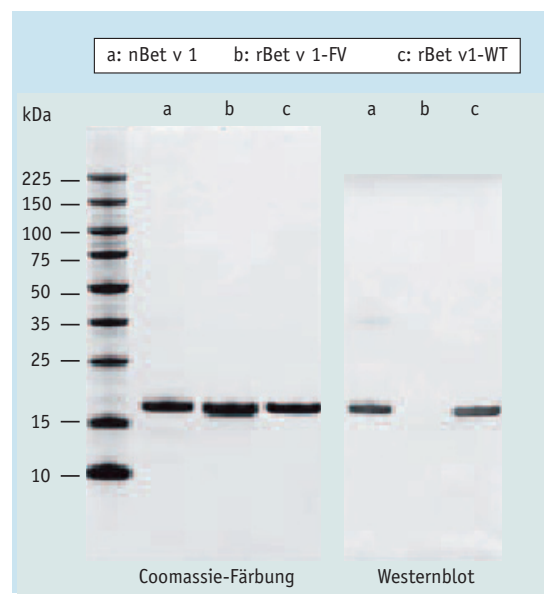

In der Gelelektrophorese mit CoomassieFärbung verhalten sich alle drei Allergene gleich, im Westernblot ist für das rekombinante Allergen mit veränderter Faltblattstruktur (rBet v 1-FV) keine Bindung an IgE-Antikörper nachweisbar.
Faltungsvariante dagegen wies hier keine Reaktion auf.

Ein Test mit basophilen Granulozyten von 19 Allergiekranken zeigte für rBet v 1-FV eine 90- bis 9.000-fache Verringerung der Aktivierungskapazität im Vergleich zu den anderen beiden Allergenen. Der Histaminfreisetzungstest ergab ebenfalls deutlich niedrigere Werte für das rekombinante Antigen mit veränderter Konformation.

Die T-Zell-Reaktivität von rBet v 1FV blieb dagegen erhalten; hier gab es keine signifikanten Unterschiede zwischen den Gruppen. Auch die Immunogenität dieses Allergens konnte im Tierversuch nachgewiesen werden, es kam zu einer klaren IgG-Antikörperbildung nach Sensibilisierung.

Fazit: Die Ergebnisse weisen darauf hin, dass das rekombinante Allergen rBet v 1-FV mit veränderter Faltstruktur hypoallergen ist, jedoch bei der gewünschten T-Zell-Reaktivität keine Einschränkungen aufweist.

af

Kahlert $\mathrm{H}$ et al. Characterization of a hypoallergenic recombinant Bet $\mathrm{v} 1$ variant as a candidate for allergen-specific immunotherapy. Int Arch Allergy Immunol 2008; 145: 193-206 